\title{
Mortality and Coronary Heart Disease in Euthyroid Patients
}

\author{
David Pereg, $\mathbf{M D}^{\mathrm{a}, \mathrm{b}, \mathrm{c}}$, Amir Tirosh, $\mathbf{M D}^{\mathrm{d}}$, Avishay Elis, MD ${ }^{\mathrm{b}, \mathrm{c}}$, Yoram Neuman, MD ${ }^{\mathrm{a}, \mathrm{c}}$, Morris \\ Mosseri, MD ${ }^{a, c}$, David Segev, MDe, Michael Lishner, MD ${ }^{b, c}$, and Doron Hermoni, MD ${ }^{c, e, f}$ \\ aDivision of Cardiology, Meir Medical Center, Kfar-Saba, Israel \\ ${ }^{b}$ Department of Internal Medicine A, Meir Medical Center, Kfar-Saba, Israel \\ 'Sackler Faculty of Medicine, Tel-Aviv University, Tel-Aviv, Israel \\ dDivision of Endocrinology, Diabetes, and Hypertension, Brigham and Women's Hospital, Harvard \\ Medical School, Boston, Mass \\ eSharon-Shomron District, Clalit Health Services, Netanya, Israel \\ fDepartment of Family Medicine, Clalit Health Services, Netanya, Israel
}

\section{Abstract}

Background-Subclinical thyroid dysfunction is associated with increased mortality and cardiovascular risk. It is unknown whether this association remains within normal thyroid function range.

Methods-The study was conducted using the computerized database of the Sharon-Shomron district of Clalit Health services. Included were subjects aged $\geq 40$ years with normal thyroid function. Patients with a history of thyroid or cardiovascular diseases or diabetes were excluded. The primary end points were all-cause mortality and the need for coronary revascularization with either percutaneous coronary intervention or coronary artery bypass grafting.

Results-The 42,149 participants were stratified into 3 groups of equal thyrotropin intervals (0.35-1.6, 1.7-2.9, and 3-4.2 mIU/L). During a mean follow-up of $4.5 \pm 2.1$ years, $4239(10.1 \%)$ participants died and 1575 (3.7\%) underwent coronary revascularization. For both women and men, the lowest mortality rates were observed in the intermediate thyrotropin group. Nevertheless, only for the low thyrotropin group, mortality risk remained significantly higher as compared with the intermediate thyrotropin group, even following multivariate model adjusted for the conventional cardiovascular risk factors, in both women (odds ratio 1.22; $95 \%$ confidence interval, 1.09-1.36 for the low thyrotropin group, compared with the intermediate group) and men (odds ratio 1.14 ; $95 \%$ confidence interval, 1.01-1.3 for the low thyrotropin group, compared with the intermediate group). There was no significant difference in the need for coronary revascularization among the 3 thyrotropin groups in both men and women.

Requests for reprints should be addressed to David Pereg, Division of Cardiology, Meir Medical Center, 59 Tchernichovsky St., KfarSaba 44281, Israel. davidpe@ post.tau.ac.il.

Conflict of Interest: None.

Authorship: All authors had access to the data and a role in writing the manuscript. 
Conclusions-Low thyrotropin level within the reference range is associated with increased risk for all-cause mortality.

\section{Keywords}

Mortality; Thyrotropin

Subclinical thyroid dysfunction refers to patients with abnormal thyrotropin (thyroidstimulating hormone [TSH]) level and a normal free thyroxine (T4) level. While subclinical hypothyroidism is reasonably common, with a prevalence of about $4.5 \%$ in adults, subclinical hyperthyroidism is less prevalent $(0.7 \%$ in adults $) .{ }^{1}$ Data about the association between subclinical thyroid disease and mortality or coronary heart disease are conflicting, and the existing studies are limited mainly by the small number of participants. ${ }^{2-7} \mathrm{~A}$ metaanalysis of 10 studies including 1491 patients with subclinical hypothyroidism and 223 patients with subclinical hyperthyroidism has suggested that both abnormalities may be associated with a modest increased risk for coronary heart disease and mortality. ${ }^{1}$ There is only a single large prospective study that has assessed the relation of normal thyroid function with coronary heart disease. ${ }^{8}$ This study included 17,311 women and 8002 men without known thyroid or cardiovascular disease or diabetes mellitus. It demonstrated that thyrotropin levels within the reference range were positively and linearly associated with coronary heart disease-related mortality in women but not in men.

In the present study, we prospectively examined the association between thyrotropin levels within the normal range and all-cause mortality and coronary heart disease in a large population-based cohort study of euthyroid patients.

\section{Methods}

\section{Study Population}

The cohort included residents of the Sharon-Shomron district who are medically insured by the Clalit Health Services (CHS), the largest health maintenance organization in Israel. CHS insures 54\% of the district's population, which is mostly urban and includes both Jews and Arabs. All medical information obtained at the primary care clinics is recorded in the CHS computerized database and can be accessed at the level of the individual patient. Each family physician is responsible for updating the computerized medical records routinely during each patient visit and following any hospital admission. The database includes a list of all diagnoses, demographic data, laboratory values, medications, and medical procedures. This database was the source of data for our study.

\section{Study Design}

Included in our study were women and men 40 years of age or older who were living in the Sharon-Shomron district and for whom thyrotropin levels were available during the year 2002. The age limit was set due to the extremely low rates of both mortality and coronary heart disease in younger participants. The specific reason that subjects had their thyrotropin assessed was not available in the database. However, all blood tests were taken from ambulatory patients in an outpatient set-up and not during hospital admission. Exclusion 
criteria included preexisting thyroid or cardiovascular disease, or diabetes mellitus at the time of thyrotropin measurement, based on information retrieved from the primary care database. Thyroid disease was defined by either the presence of a diagnosis of hypo- or hyperthyroidism in the medical record, treatment with levothyroxine or antithyroid medications, or with documented abnormal thyrotropin or T4 levels. Cardiovascular disease was defined as the presence of either of the following diagnoses: coronary artery disease, ischemic or hemorrhagic stroke, transient ischemic attack, congestive heart failure, or peripheral vascular disease. Participants were defined as having diabetes if the diagnosis appeared in their medical records or if antidiabetic medications were prescribed.

Hypertension was considered when such a diagnosis appeared on the patient's diagnosis list or when chronic treatment with antihypertensive medications was administered. Body mass index (BMI) was calculated as the weight in kilograms divided by the square of the height in meters. Height and weight were measured at the primary care clinics.

\section{Follow-up and Study Endpoints}

Participants were followed from their initial thyrotropin measurement in 2002 until censorship for outcomes or October 2010, whichever came first. The 2 primary end points of our study were all-cause mortality and coronary revascularization with either percutaneous coronary interventions, or coronary artery bypass grafting surgery. Mortality data were collected from the National Social Security records. Information about the specific causes of death was not available to us. All revascularization procedures are performed at the regional hospitals and are automatically recorded in the CHS database, resulting in an accurate and reliable outcome determination.

\section{Laboratory Analysis}

Serum thyrotropin level determinations were performed using a continuous random access analyzer (Immulite 2000; Diagnostic Products Corp., Los Angeles, Calif) and an immunoassay (ADVIA centaur; Bayer Health Care LLC, Diagnostics Division, Tarrytown, NY) apparatus. The reference range for thyrotropin is $0.35-4.2 \mathrm{mIU} / \mathrm{L}$, and for free $\mathrm{T} 4$ is $0.8-2 \mathrm{ng} / \mathrm{dL}$.

\section{Ethical Considerations}

The study was approved by the local institutional ethics committee in keeping with the principles of the Declaration of Helsinki. In accordance with Ministry of Health regulations, the institutional ethics committee did not require written informed consent because data were collected anonymously from the computerized medical files, with no active participation of patients.

\section{Statistical Analysis}

The study subjects were stratified into 3 groups of equal thyrotropin intervals (0.35-1.6, 1.7-2.9, and 3-4.2 mIU/L) based on their thyrotropin level determined at enrollment. The reasoning for dividing the study population into tertiles was the very narrow Gaussian distribution of thyrotropin in the study population. The baseline clinical characteristics of these patient groups were compared using analysis of variance test for continuous variables 
and chi-squared test for nonparametric variables. Logistic regression analysis was used to estimate odds ratios and 95\% confidence intervals (CIs) for all-cause mortality and for coronary revascularization (percutaneous coronary interventions or coronary artery bypass grafting) according to the 3 groups of thyrotropin. This analysis was adjusted for age (continuous variable), sex, low-density lipoprotein (LDL) and high-density lipoprotein (HDL) cholesterol (continuous), BMI (continuous), and smoking. For all variables, the most recent values were used. Because sex is a major determinant of the incidence of both thyroid and cardiovascular diseases, ${ }^{8}$ all analyses were conducted separately for men and women. Statistical analysis was performed with the use of SPSS statistical software for Windows, version 17.0 (IBM Corporation, Armonk, NY).

\section{Results}

The database included 79,433 patients aged 40 years or above for whom thyrotropin level was documented during the year 2002. Of them, 37,284 patients were excluded due to preexisting thyroid disease, diabetes mellitus, or cardiovascular disease, leaving a total of 42,149 subjects, including 28,446 (67.5\%) women, available for analysis. The mean age and BMI were $57.5 \pm 12.4$ years and $27.7 \pm 4.8$, respectively. Baseline characteristics stratified by thyrotropin level within the normal range as measured at baseline (low, intermediate, and high thyrotropin) are described in Table 1. Overall, for both men and women, as thyrotropin levels were higher, participants were older and more frequently hypertensive, and had higher BMI, cholesterol, and triglycerides levels.

\section{Normal Thyroid Function and Mortality}

During a mean follow-up of $4.5 \pm 2.1$ years, 4239 (10\%) participants died and 1575 (3.7\%) underwent coronary revascularization. Odds ratios for all-cause mortality according to thyrotropin levels in men and women are presented in Table 2. For both women and men, significantly lower mortality rates were observed in the intermediate thyrotropin group. Allcause mortality rates in the low, intermediate, and high thyrotropin groups among women were $9.8 \%, 7.8 \%$, and $8.8 \%$, and among men were $13.2 \%, 11.4 \%$, and $14.9 \%$, respectively. In a multivariate model adjusted for age, LDL and HDL cholesterol, triglyceride levels, BMI, smoking, and hypertension, higher thyrotropin level within the normal range (TSH 3.0-4.2 mIU/L) was not associated with increased mortality rates as compared with the intermediate group. Nevertheless, for the low thyrotropin group (TSH 0.35-1.6 mIU/L), mortality risk remained significantly higher as compared with the intermediate thyrotropin group, even in the multivariate model adjusted for all the above-mentioned parameters, in both women (odds ratio 1.22; 95\% confidence interval, 1.09-1.36 for the low thyrotropin group, compared with the intermediate group) and men (odds ratio 1.14; $95 \%$ confidence interval, 1.01-1.3, for the low thyrotropin group, compared with the intermediate group).

\section{Normal Thyroid Function and Coronary Heart Disease}

Odds ratios for coronary revascularization according to thyrotropin levels in women and men are presented in Table 3 . There was no significant difference in the need for coronary revascularization among the 3 thyrotropin groups in both men and women. Coronary revascularization rates in the low, intermediate, and high thyrotropin groups among women 
were $1.8 \%, 1.9 \%$, and $1.9 \%$, and among men $7.5 \%, 7.9 \%$, and $7.8 \%$, respectively. The risks for coronary revascularization of the 3 groups remained similar following multivariate adjustments.

\section{Discussion}

In this large cohort study of Israeli men and women, we have demonstrated that both low and high thyrotropin levels within the reference range are associated with increased all-cause mortality in both sexes. Nevertheless, only in the low thyrotropin group did this increased mortality risk remain statistically significant following multivariate analysis. Regarding coronary heart disease, we didn't find any association of thyrotropin levels within the normal range and coronary revascularization.

Only a single large prospective study has investigated the association between thyrotropin within the reference range and coronary heart disease. ${ }^{8}$ The study included 15,462 women and 7447 men with normal thyrotropin levels that were divided into 3 groups. Patients with any thyroid or cardiovascular disease or diabetes were excluded. Similar to our findings, patients in the middle thyrotropin group were younger. Furthermore, higher thyrotropin level within the reference range was associated with more cardiovascular risk factors, including higher BMI and blood pressure and poor lipid profile. However, while in both studies the percentage of current smokers tended to be inversely associated with thyrotropin levels, the rate of smokers in our study was much lower for both men and women. The study demonstrated a linear association between thyrotropin and fatal coronary heart disease only in women. However, data about nonfatal coronary heart disease or all-cause mortality were not available. Our study did not include data about the cause of death. However, because the 3 thyrotropin groups had similar coronary revascularization rates and, considering the better cardiovascular risk profile in the low thyrotropin group, it is unlikely that the increase in allcause mortality in this group is due to coronary heart disease. The shorter mean follow-up time ( 4.5 vs. 8.3 years) and the lower percentage of smokers in our study may partially explain the lack of increased coronary heart disease in the high thyrotropin group despite more cardiovascular risk factors in these patients.

The association between low thyrotropin within the reference range and increased all-cause mortality may have several possible explanations. A number of studies have reported that both overt and subclinical hyperthyroidism may be associated with increased cardiovascular and all-cause mortality. ${ }^{1,9,10}$ Several mechanisms for the increased mortality have been suggested, including increased systemic inflammation and hypercoagulability, ${ }^{11}$ enhanced atherosclerosis, ${ }^{12}$ atrial fibrillation, ${ }^{13}$ and left ventricular hypertrophy. ${ }^{14}$ Nevertheless, interpretation of these studies is hampered by the low number of subjects and the lack of multivariate adjustment to many possible confounding factors. It may be reasonable to assume that patients with low TSH levels within the normal range resemble those with subclinical hyperthyroidism and therefore, may have the same mechanisms for increased mortality. Because low thyrotropin levels within the normal range are far more common than subclinical and overt hyperthyroidism, our results are complementary to the previous studies and support the association of subclinical hyperthyroidism with adverse outcome. On the other hand, it is possible that the low thyrotropin itself is not a direct cause for the increased 
mortality but rather a marker for high-risk patients due to other comorbidities that were not included in our study. The clinical significance of our study is the identification of a population at significantly higher mortality risk. However, further investigation is needed for better understanding of this association before diagnostic or therapeutic recommendations can be made.

Although our study is the first to investigate the association of thyrotropin levels within the normal range and all-cause mortality and coronary heart disease in a relatively healthy population, it has several limitations that warrant consideration. First, the evaluation of thyrotropin levels was based on a single measurement and therefore, within-person variation may be a potential source for measurement error. Second, because the study population was not proactively screened, and thyrotropin levels were available only for those who attended their primary care providers, there may have been a selection bias. Third, the primary end points included all-cause mortality, and we did not have data about specific causes of death, most importantly regarding cardiovascular death. The other end point was the need for coronary revascularization. This outcome was chosen because medical procedures, including percutaneous coronary intervention and coronary artery bypass grafting, are automatically recorded in the CHS computerized database, independent of the primary care physician follow-up, making coronary revascularization the most reliable outcome for coronary heart disease. While our study did not include any data about other cardiovascular diseases, coronary revascularization is a highly sensitive and specific cardiovascular end point that covers the entire clinical spectrum of significant coronary heart disease. Finally, the possibility that increased death rates in the lower thyrotropin group have undermined the potential association between low TSH level and cardiovascular outcome as estimated by revascularization events cannot be ruled out.

Our study adds to the available literature by focusing on the normal thyrotropin range and by demonstrating that a single measurement of low-serum thyrotropin in euthyroid men and women aged 40 years or older is associated with increased mortality from all causes.

\section{Acknowledgments}

We thank the data center team at the Sharon-Shomron district for their help in data retrieving.

Funding: None.

\section{References}

1. Ochs N, Auer R, Bauer DC, et al. Meta-analysis: subclinical thyroid dysfunction and the risk for coronary heart disease and mortality. Ann Intern Med. 2008; 148:832-845. [PubMed: 18490668]

2. Cappola AR, Fried LP, Arnold AM, et al. Thyroid status, cardiovascular risk, and mortality in older adults. JAMA. 2006; 295:1033-1041. [PubMed: 16507804]

3. Razvi S, Weaver JU, Vanderpump MP, Pearce SH. The incidence of ischemic heart disease and mortality in people with subclinical hypothyroidism: reanalysis of the Whickham Survey cohort. J Clin Endocrinol Metab. 2010; 95:1734-1740. [PubMed: 20150579]

4. Imaizumi M, Akahoshi M, Ichimaru S, et al. Risk for ischemic heart disease and all-cause mortality in subclinical hypothyroidism. J Clin Endocrinol Metab. 2004; 89:3365-3370. [PubMed: 15240616]

5. Rodondi N, Aujesky D, Vittinghoff E, et al. Subclinical hypothyroidism and the risk of coronary heart disease: a meta-analysis. Am J Med. 2006; 119:541-551. [PubMed: 16828622] 
6. Rodondi N, Newman AB, Vittinghoff E, et al. Subclinical hypothyroidism and the risk of heart failure, other cardiovascular events, and death. Arch Intern Med. 2005; 165:2460-2466. [PubMed: 16314541]

7. Singh S, Duggal J, Molnar J, et al. Impact of subclinical thyroid disorders on coronary heart disease, cardiovascular and all-cause mortality: a meta-analysis. Int J Cardiol. 2008; 125:41-48. [PubMed: 17434631]

8. Asvold BO, Bjøro T, Nilsen TI, et al. Thyrotropin levels and risk of fatal coronary heart disease: the HUNT study. Arch Intern Med. 2008; 168:855-860. [PubMed: 18443261]

9. Parle JV, Maisonneuve P, Sheppard MC, et al. Prediction of all-cause and cardiovascular mortality in elderly people from one low serum thyrotropin result: a 10-year cohort study. Lancet. 2001; 358:861-865. [PubMed: 11567699]

10. Osman F, Franklyn JA, Holder RL, et al. Cardiovascular manifestations of hyperthyroidism before and after antithyroid therapy: a matched case-control study. J Am Coll Cardiol. 2007; 49:71-81. [PubMed: 17207725]

11. Dorr M, Robinson DM, Wallaschofski H, et al. Low serum thyrotropin is associated with high plasma fibrinogen. J Clin Endocrinol Metab. 2006; 91:530-534. [PubMed: 16303831]

12. Volzke H, Robinson DM, Schminke U, et al. Thyroid function and carotid wall thickness. J Clin Endocrinol Metab. 2004; 89:2145-2149. [PubMed: 15126533]

13. Sawin CT, Geller A, Wolf PA, et al. Low serum thyrotropin concentrations as a risk factor for atrial fibrillation in older persons. N Engl J Med. 1994; 331:1249-1252. [PubMed: 7935681]

14. Dorr M, Wolff B, Robinson DM, et al. The association of thyroid function with cardiac mass and left ventricular hypertrophy. J Clin Endocrinol Metab. 2005; 90:673-677. [PubMed: 15522926] 


\section{Clinical Significance}

- $\quad$ A single measurement of low-serum thyrotropin in euthyroid men and women aged 40 years or older is associated with increased mortality from all causes.

- There was no association between normal thyrotropin levels and coronary heart disease. 
Table 1

Baseline Characteristics of the Study Population

\begin{tabular}{|c|c|c|c|c|}
\hline \multirow[b]{2}{*}{ Characteristics } & \multicolumn{4}{|c|}{ Thyrotropin mIU/L } \\
\hline & $0.35-1.6$ & $1.7-2.9$ & $3-4.2$ & $P$ Value \\
\hline \multicolumn{5}{|l|}{ Women } \\
\hline Participants, $\mathrm{n}$ & 10,738 & 12,343 & 5365 & \\
\hline Thyrotropin, median, mIU/mL & 1.21 & 2.22 & 3.49 & \\
\hline Age (SD), years & $57.2(13)$ & $56.9(12)$ & $58.2(12)$ & $<.001$ \\
\hline Current smokers, $\%$ & 21.6 & 21.2 & 20.8 & .46 \\
\hline \multicolumn{5}{|l|}{ Lipids, mean (SD), mg/dL } \\
\hline Total cholesterol & $214(38)$ & $217(39)$ & $218(40)$ & $<.001$ \\
\hline LDL cholesterol & $131(32)$ & $133(32)$ & $134(33)$ & $<.001$ \\
\hline HDL cholesterol & $56(13)$ & $56(13)$ & $56(13)$ & .56 \\
\hline Triglycerides & $129(71)$ & $135(80)$ & $139(45)$ & $<.001$ \\
\hline Hypertension & 41 & 42 & 42 & .049 \\
\hline BMI, mean (SD) & $27.6(5)$ & $27.7(5)$ & $28(5)$ & .04 \\
\hline \multicolumn{5}{|l|}{ Men } \\
\hline Participants, $\mathrm{n}$ & 6330 & 5766 & 1607 & \\
\hline Thyrotropin, median, mIU/mL & 1.22 & 2.16 & 3.43 & \\
\hline Age (SD), years & $58(12)$ & $58(12)$ & $59(12)$ & $<.001$ \\
\hline Current smokers, $\%$ & 43 & 39 & 38 & $<.001$ \\
\hline \multicolumn{5}{|l|}{ Lipids, mean (SD), mg/dL } \\
\hline Total cholesterol & $207(39)$ & $208(40)$ & 209 (39) & .046 \\
\hline LDL cholesterol & $129(32)$ & $131(32)$ & $131(32)$ & .82 \\
\hline HDL cholesterol & $47(11)$ & $46(10)$ & $46(11)$ & $<.001$ \\
\hline Triglycerides & $152(99)$ & $163(115)$ & $166(124)$ & $<.001$ \\
\hline Hypertension & 43 & 45 & 48 & .004 \\
\hline BMI, mean (SD) & $27.5(4)$ & 27.7 & 27.9 & .001 \\
\hline
\end{tabular}

$\mathrm{BMI}=$ body mass index $; \mathrm{HDL}=$ high-density lipoprotein $\mathrm{LDL}=$ low-density lipoprotein. 
Table 2

Relative Risk for All-cause Mortality According to Thyrotropin Levels

\begin{tabular}{|c|c|c|c|c|}
\hline & \multicolumn{4}{|c|}{ Thyrotropin mIU/L } \\
\hline & $0.35-1.6$ & 1.7-2.9 & 3-4.2 & $P$ Value \\
\hline \multicolumn{5}{|l|}{ Women } \\
\hline Mortality, \% (n) & $9.8(1050)$ & $7.8(968)$ & $8.8(474)$ & $<.0001$ \\
\hline Mortality (n/1000 person-years) & 12.2 & 9.8 & 11.1 & \\
\hline Unadjusted risk ratio & $1.27(1.16-1.34)$ & 1 & $1.14(1.01-1.28)$ & \\
\hline \multicolumn{5}{|l|}{ Adjusted risk ratio } \\
\hline Age & $1.23(1.10-1.36)$ & 1 & $1.02(0.89-1.17)$ & \\
\hline Age, LDL, HDL, Total cholesterol, TG, BMI, smoking, hypertension & $1.22(1.09-1.36)$ & 1 & $1.03(0.9-1.18)$ & \\
\hline \multicolumn{5}{|l|}{ Men } \\
\hline Mortality, \% (n) & $13.2(835)$ & $11.4(655)$ & $14.9(239)$ & $<.0001$ \\
\hline Mortality (n/1000 person-years) & 16.5 & 14.2 & 18.6 & \\
\hline Unadjusted risk ratio & $1.19(1.06-1.32)$ & 1 & $1.36(1.16-1.6)$ & \\
\hline \multicolumn{5}{|l|}{ Adjusted risk ratio } \\
\hline Age & $1.16(1.02-1.31)$ & 1 & $1.14(0.95-1.37)$ & \\
\hline Age, LDL and HDL cholesterol, TG, BMI, smoking, hypertension & $1.14(1.01-1.3)$ & 1 & $1.18(0.98-1.43)$ & \\
\hline
\end{tabular}

$\mathrm{BMI}=$ body mass index $; \mathrm{HDL}=$ high-density lipoprotein $; \mathrm{LDL}=$ low-density lipoprotein $\mathrm{TG}=$ triglyceride. 
Table 3

Relative Risk for Coronary Revascularization, According to Thyrotropin Levels

\begin{tabular}{|c|c|c|c|c|}
\hline & \multicolumn{4}{|c|}{ Thyrotropin mIU/L } \\
\hline & $0.35-1.6$ & $1.7-2.9$ & $3-4.2$ & $P$ Value \\
\hline \multicolumn{5}{|l|}{ Women } \\
\hline Coronary revascularization, $\%(\mathrm{n})$ & $1.8(189)$ & $1.9(231)$ & $1.9(100)$ & .8 \\
\hline Coronary revascularization ( $\mathrm{n} / 1000$ person-years) & 2.2 & 2.3 & 2.3 & \\
\hline Unadjusted risk ratio & $0.94(0.77-1.14)$ & 1 & $0.99(0.79-1.3)$ & \\
\hline \multicolumn{5}{|l|}{ Adjusted risk ratio } \\
\hline Age & $0.92(0.76-1.12)$ & 1 & $0.96(0.75-1.2)$ & \\
\hline Age, LDL, HDL, TG, BMI, smoking, hypertension & $0.95(0.78-1.2)$ & 1 & $0.94(0.74-1.2)$ & \\
\hline \multicolumn{5}{|l|}{ Men } \\
\hline Coronary revascularization, \% (n) & $7.5(477)$ & $7.9(453)$ & $7.8(125)$ & .8 \\
\hline Coronary revascularization ( $\mathrm{n} / 1000$ person-years) & 9.4 & 9.8 & 9.7 & \\
\hline Unadjusted risk ratio & $0.96(0.84-1.1)$ & 1 & $0.99(0.8-1.2)$ & \\
\hline \multicolumn{5}{|l|}{ Adjusted risk ratio } \\
\hline Age & $0.95(0.83-1.09)$ & 1 & $0.97(0.79-1.2)$ & \\
\hline Age, LDL and HDL cholesterol, TG, BMI, smoking, hypertension. & $0.98(0.85-1.2)$ & 1 & $0.94(0.76-1.16)$ & \\
\hline
\end{tabular}

$\mathrm{BMI}=$ body mass index $; \mathrm{HDL}=$ high-density lipoprotein $; \mathrm{LDL}=$ low-density lipoprotein $\mathrm{TG}=$ triglyceride. 